\title{
Amalgam yüzeyine uygulanan farklı yüzey işlemlerinin ve farklı adeziv sistemlerinin ortodontik amaçlı molar tüplerin bağlanma dayanımları üzerine etkilerinin değerlendirilmesi
}

\author{
Ahmet Ertan Soğancı ${ }^{\alpha}$, Said Karabekiroğlu ${ }^{\beta}$, Zeliha Bektaş ${ }^{\alpha}$, Merve Gürses ${ }^{\beta}$, Nimet Ünlür
}

Selcuk Dent J, 2017; 4: 134-138 (Doi: 10.15311/selcukdentj.297907)

Başvuru Tarihi: 17 Mart 2017 Yayına Kabul Tarihi: 17 Kasım 2017

öz

Amalgam yüzeyine uygulanan farklı yüzey işlemlerinin ve farklı adeziv sistemlerinin ortodontik amaçlı molar tüplerin bağlanma dayanımları üzerine etkilerinin değerlendirilmesi

Amaç: Bu araştırmanın amacı, kumlama, elmas frez ve Er-YAG lazer ile pürüzlendirilmiş farklı amalgam yüzeylerine farklı yapıştırma sistemleri ile yapıştııılmış ortodontik molar tüplerin bağlanma dayanımlarının karşılaştııııasıdır.

Gereç ve Yöntemler: Kendiliğinden sertleşen akrilik ile imal edilen 60 kalıpta molar tüpler için standart boşluklar hazırlanıp, amalgam ile dolduruldu. Numuneler, rastgele yüzey pürüzlendirmesi için 20 örnekten oluşan 3 gruba ayrıldı ve ayrıca 2 farklı yapıştırıcıya göre 2 alt gruba bölündü. 1. Grupta örnek yüzeyleri elmas frezle, 2. grupta aliminyum oksit tozu ile 3 . grupta ise Er-YAG lazer ünitesi ile pürüzlendirildi. Molar tüplerinin bütün gruplardaki amalgam yüzeylerine bağlanması için 2 farklı yapıştırma sistemi uygulandı. Universal test makinesi kullanılarak makaslama kuvveti uygulandı. Molar tüplerinin örneklerden söküldüğü andaki kuvvetler kaydedildi.

Bulgular: Ortodontik molar tüplerin, amalgam yüzeylerine bağlanma dayanımı değerleri karşılaştıııldığında farklı pürüzlendirme yöntemlerine ve 2 farklı yapıştırma sistemine göre istatistiksel olarak anlamlı fark bulunmadı.

Sonuç: Amalgam yüzeyindeki tüm yüzey pürüzlendirme işlemlerinin, bu çalışmada kullanılan 2 farklı yapıştırma sistemi ile yapıştırıan ortodontik molar tüplerin, tüm gruplarda benzer bağlanma dayanımına sahip olduğu saptanmışıı.

\section{ANAHTAR KELIMELER}

Adheziv sistemler, amalgam, bağlanma dayanımı, Er-YAG lazer, Ortodonti

Daimi birinci molar dişler, ağız ortamına ilk süren dişlerdendir ve hem karma hem de daimi dentisyon için büyük öneme sahiptirler. Bu dönemde, tam olarak ağız sağlığı bilinci gelişmemiş çocuklarda bu dişlerin bakımı inmal edildiğinde erken kayıplar veya dişin büyük bir kısmını içeren restorasyonlar kaçınılmaz olur. Bu dişlerin restorasyonlarında, klinik kullanım kolaylığı, aşınma

\section{ABSTRACT}

Evaluation of bond strength of orthodontic molar tubes bonded to amalgam with different surface treatment procedures and different adhesive systems

Background: The aim of this study is to investigate the shear bonding strength of orthodontic molar tubes bonded to the different amalgam surfaces, roughened by sandblasting, diamond bur and Er-YAG laser with different adhesive systems.

Methods: 60 acrylic molds were fabricated with self-cure resin. In acrylic molds, standard cavities were prepared for molar tubes and amalgam was condensed into the cavities. The specimens were randomly divided into 3 groups of 20 pieces for surface roughening, and divided to 2 subgroups according to 2 different bonding adhesives. In group 1 surfaces were roughened with a diamond bur, in group 2 surfaces were sandblasted, in group 3 Er-YAG laser unit was used for surface treatment. 2 different adhesive systems were applied for bonding of molar tubes to the all groups of amalgam surfaces. Debonding was performed with a shearing force using universal testing machine.

Results: No statistically significant difference was determined between adhesive strength and roughening parameters on bonding strength values of orthodontic molar tubes to amalgam surfaces.

Conclusion: It was determined that all surface roughening procedures on the amalgam surface and 2 different adhesive systems used in this study have similar bonding strength in the bonding of orthodontic molar tubes to the all groups.

\section{KEYWORDS}

Adhesive systems, amalgam, bond strength, Er-YAG laser, orthodontics

dayanımının yüksek oluşu, mikro sızıntı riskini azaltması, çiğneme kuvvetlerine karşı dayanıklı olması ve rahat ulaşılabilmesi gibi nedenlerle geçmişte sıklıkla ve hala günümüzde amalgam dolgu materyali tercih edilmektedir. ${ }^{2}$

\footnotetext{
${ }^{\alpha}$ Necmettin Erbakan Üniversitesi Diş Hekimliği Fakültesi Ortodonti Anabilim Dalı, Konya

${ }^{\beta}$ Necmettin Erbakan Üniversitesi Diş Hekimliği Fakültesi Restoratif Diş Tedavisi Anabilim Dalı, Konya

${ }^{v}$ Selçuk Üniversitesi Diş Hekimliği Fakültesi Restoratif Diş Tedavisi Anabilim Dalı, Konya
} 
Genç yetişkin ortodonti hastalarının bir kısmında posterior dişlerinin bukkal yüzeylerinde amalgam restorasyonlar mevcuttur. ${ }^{13}$ Ortodontik braketlerin restorasyon materyalleri ile bağlantısı mineye bağlantısına göre oldukça düşüktür. Ortodontik tedavi gören yetişkin hastaların sayısının giderek artması göz önüne alındığında, ortodontik braketlerin amalgama bağlanması klinik açıdan oldukça önem arz etmektedir. ${ }^{2} \mathrm{Bu}$ klinik problem nedeniyle amalgam ve braket bağlantısı ile ilgili araştırmalar yapılmış ve bu araştırmaların sonucunda amalgam bağlantısının geliştirilmesi için farklı prosedürlere intiyaç olduğu bildirilmiştir. ${ }^{6}$ Farklı adeziv sistemler kullanılarak uygulanan, elmas frezle pürüzlendirme ve kumlama gibi mekanik yüzey işlem prosedürlerinin etkili bağlantının gerçekleştirilmesine olumlu katkı sağlayabileceği bazı literatürlerde rapor edilmiştir.2, 10, 13

Son zamanlarda Er-YAG lazer sistemleri de diş hekimliğinde yüzey işlemleri için yeni bir yöntem olarak büyük ilgi çekmektedir. Daha önceki çalışmalarda $\mathrm{Er}, \mathrm{Cr}$ YSGG lazer ve asitleme işlemi ile pürüzlendirilen minedentin yüzeyleri karşılaştırılmıştır. ${ }^{7}{ }^{11}$ Restoratif materyal üzerindeki etkileri tam olarak bilinmemesine rağmen sulu veya susuz kullanılan Er-YAG lazerlerin amalgam üzerinde ablasyon ile $100 \mu \mathrm{m}$ çapında kraterler oluşturduğu gözlenmiştir. Bu oluşan girintili-çıkıntılı yüzey sayesinde ortodontik braketlerin amalgam yüzeylerine bağlanma direncinin olumlu etkileneceği düşünülmektedir. ${ }^{9,3}$

$\mathrm{Bu}$ in vitro çalışmanın amacı, kumlama, elmas frez ve ErYAG lazer ile pürüzlendirilen amalgam yüzeylerine farklı adeziv sistemler kullanılarak yapıştırılan ortodontik molar tüplerin amalgam yüzeye bağlanma dayanımını incelemek ve klinikte amalgam yüzeye en etkili bağlanma dayanımı sergileyebilecek seçeneği tespit edebilmektir.

\section{GEREÇ VE YÖNTEM}

Bu çalışma için her biri $5 \mathrm{~cm}$ uzunluğunda ve $2,5 \mathrm{~cm}$ çapında olan plastik kalıplar hazırlandı ve bu kalıplara otopolimerizan akrilik(Self Curing, Vortex, Netherland) yerleştirilerek 60 adet akrilik kalıp elde edildi. Akrilik

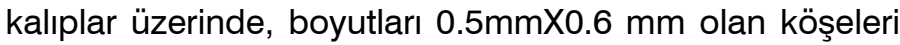
yuvarlatılmış dikdörtgen kaviteler açılarak, molar tüplerin bağlanacağı bu kavitelere, Gama 2 fazı içermeyen gümüş amalgam materyali(GK-110 AT \& M BiomaterialsCo. Ltd.) ile restorasyonlar yapıldı. El aletleriyle cilalama işlemi tamamlanan örnekler, distile su içerisinde, oda sıcaklığında 24 saat bekletildi. Daha sonra gri ve yeşil renkli amalgam polisaj frezleri(Politip-F, Politip-P, Ivoclar, Vivadent, Liechtenstein) ile bitim ve polisaj işlemi uygulandı. Örnekler kullanılan pürüzlendirme yöntemine göre her grupta 20 adet örnek olmak üzere 3 gruba ayrıldı; bu 3 gruptaki örnekler, uygulanacak 2 farklı adeziv sistemine göre 2 alt gruba ayrıldı.

\section{Amalgam yüzey işlemleri}

Çalışmada, amalgam yüzeylerine ortodontik molar tüplerin bağlanma dayanımını arttırmak için 3 farklı yüzey düzenleme metodu kullanıldı:

(1) Elmas frezle pürüzlendirme: 20 adet amalgam örneğin adeziv rezin uygulanacak olan yüzeyi klinik uygulamada yapıldığı şekilde elmas silindirik frez (yeşil kuşak No:12C, SWS Dental, Türkiye) kullanılarak su soğutması altında 3 sn süreyle pürüzlendirildi. Her 5 örnekten sonra kullanılan frez değiştirildi.

(2) Kumlama (Al2O3 tozları ile): Amalgam yüzeyleri 50 mikron $(\mu)$ boyutundaki aliminyum oksit partikülleri ile 4,1 bar basınç altında $10 \mathrm{~mm}$ mesafeden 3 sn süre ile kumlandı (Microblaster, Dento-prep, DentolMicroblaster. Denmark).

(3) Lazer ile pürüzlendirme(Er-YAG Fotona LightWalker, Model M021-5 AF/1, Slovenia): Bu lazer sistemi $2.78 \mu \mathrm{m}$ dalga boyunda, 140-200 $\mu$ s lik periyotlarla vuruş yapan fotonlar yaymaktadır. Bu cihazın çıkış gücü 0 ile $6 \mathrm{~W}$ arasında değişim göstermektedir. 1W lazer gücü (\%20 airlevel, \%10 waterlevel) yapılan bir pilot çalışmada optimum olarak belirlendi. Çapı $0.282 \mathrm{~mm}^{2}$ olan ışın huzmesi SSP (supershortpulse) modunda, ışın yüzeye dik gelecek şekilde yaklaşık $1 \mathrm{~mm}$ uzaklıkta 5 sn boyunca amalgam yüzeylere uygulandı.

\section{Adeziv sistemlerin uygulanışı}

Pürüzlendirme işlemleri tamamlanan amalgam yüzeylerine 2 farklı tip adeziv sistem (Transbond XT ve Clearfil Universal Bond) üretici firmanın talimatları doğrultusunda uygulandı. Tek tip ortodontik kompozit rezin materyal, (Transbond $\mathrm{XT}$, ortodontik kompozit rezin, 3M Unitek, Monrovia, CA, ABD) kullanılarak paslanmaz çelik molar tüpler (American Orthodontics WI, ABD) amalgam restorasyonlar üzerine yerleştirildi.

(1-a, 2-a, 3-a); 3 farklı metotla pürüzlendirilen 30 amalgam örneği hava-su spreyi ile kurutuldu. Transbond XT bonding ajanı, (3M Unitek, Monrovia, $\mathrm{CA}, \mathrm{ABD}$ ) hazırlanmış amalgam yüzeyine ince bir tabaka halinde uygulandı ve 10 saniye boyunca LED ışık cihazı (Hilux LEDMAX4, Benlioğlu Dental, Ankara, Türkiye) kullanılarak polimerize edildi. Cihazın ışık yoğunluğu her 10 dakikada bir ölçülerek daima aynı şiddette güç kullanımı sağlandı. Polimerizasyon işlemi boyunca cihazın ürettiği güç hiçbir zaman $400 \mathrm{~mW} / \mathrm{cm}^{2}$ 'nın altına inmedi.

(1-b, 2-b, 3-b); ilk gruptaki gibi 3 farklı metot ile pürüzlendirilen 30 amalgam örneği hava-su spreyi ile kurutuldu. Clearfil Universal Bond kitinin ilk olarak Alloyprimeri (Clearfil Universal Bond, 
Kuraray) hazırlanmış amalgam yüzeyine ince bir tabaka halinde 5 saniye uygulandı. Ardından Clearfil Universal Bond'un adeziv rezini ince bir tabaka halinde primer uygulanmış amalgam yüzeyine uygulanarak 5 saniye boyunca LED ışık cihazı (Hilux LEDMAX4, Benlioğlu Dental, Ankara, Türkiye) kullanılarak polimerize edildi.

Transbond XT, ortodontik kompozit rezin(adeziv pasta) ise bütün amalgam grupların yüzeyine yapıştırılacak olan molar tüplerin zeminine yerleştirildi. Daha sonra molar tüpler, amalgam yüzeyinde uygun pozisyona getirilerek hafifçe bastırıldı ve molar tüpün etrafına taşan artık kompozit rezin keskin bir küret yardımıyla temizlendi. Kompozit, 20 saniye insizal yönden ve 20 saniye gingival yönden olmak üzere toplam 40 snLED ışık cihazı ile polimerize edildi.

Dijital kumpas yardımıyla (Masel Orthodontics, 2701 Bartram Road, Bristol, PA, ABD) braketlerin yüzey alanları hesaplandı. Hazırlanan örnekler 48saat boyunca \% 100 nemli ortamda $37{ }^{\circ} \mathrm{C}$ 'deki etüvde bekletildikten sonra Micro 500Universal Test Cihazına (TestomelıicCo Ltd., U.K.) bağlandı ve örnekler 1 $\mathrm{mm} / \mathrm{dk}^{\prime}$ 'ık hız ile makaslama kuvvetleri uygulanarak kırıldı. Kırılma esnasında oluşan makaslama kuvvetleri, Newton olarak ölçüldü. Bağlanma alanı tespit edildikten sonra veriler megapaskala (MPa) dönüştürülerek, bağlanma dayanım değerleri hesaplandı

Molar tüpler amalgam yüzeylerinden koptuktan sonra örneklerin yüzeyi 20x büyütme değerine sahip Stereomikroskop(Discovery V8 Stereo, Carl Zeiss Micro Imaging $\mathrm{GmbH}$, Göttingen, Almanya) yardımıyla incelendi. Amalgam yüzeylerin üzerinde kalan artık adeziv, Årtun ve Bergland tarafından tanımlanan "Artık Adeziv Endeksi" (AAE) (AdhesiveRemnant Index=ARI) kullanilarak sınıflandırıldı.

Skor 0: Diş yüzeyinde hiç adeziv kalmamıştır (<10\%),

Skor 1: Diş yüzeyinde \%50'dendaha az adeziv kalmıştır. Karma kopma oluşmuştur.

Skor 2: Diş yüzeyinde $\% 50$ 'den daha fazla adezivkalmıştır.

Skor 3: Tüm adeziv diş yüzeyinde kalmıştır (>90\%)

Elde edilen veriler simetrik dağılım gösterdiği için gruplar arasındaki fark, One way ANOVA ve bunu takiben Tukey HSD post-hoc testleri ile incelenmiştir (SPSS 17.0 for Windows).

\section{BULGULAR}

Gruplara ait ortalama bağlanma dayanım değerleri Tablo 1'de sunulmuştur. Örneklerden elde edilen bağlanma dayanım değerleri arasında yapılan istatistik analiz sonuçlarına göre; amalgam yüzeylerine ortodontik molar tüplerin bağlanma dayanım değerleri üzerine adeziv tipinin ve pürüzlendirme parametrelerinin istatistiksel olarak anlamlı bir etkileri bulunamamıştır $(p>0,05)$. Adeziv tipinden bağımsız olarak lazer gruplarında en yüksek bağlanma dayanım değerleri elde edilmiş olmasına rağmen, bu değerler ile frez ve kumlama yöntemlerinde elde edilen değerler arasında istatistiksel olarak anlamlı bir farklılık olmadığı görülmektedir $(p>0,05)$.

\section{Tablo 1.}

\section{Grupların bağlanma dayanım değerlerinin ortalama ve standart sapmaları}

\begin{tabular}{|cccccc|} 
Adezivler & $\begin{array}{c}\text { Pürüzlendirme } \\
\text { tipi }\end{array}$ & $\begin{array}{c}\text { Mean } \\
(\mathbf{M P a})\end{array}$ & S.D. & ANOVA & Tukey \\
\hline $\begin{array}{c}\text { Transbond } \\
\text { XT }\end{array}$ & Frez & 5.86 & 1.24 & $\mathrm{~A}$ \\
& Kumlama & 6.35 & 1.40 & $\mathrm{~A}$ \\
& Lazer & 7.30 & 1.65 & $\mathrm{p}>0.05$ & $\mathrm{~A}$ \\
\hline Clearfil & Frez & 5.48 & 1.09 & & $\mathrm{~A}$ \\
Universal & Kumlama & 5.95 & 1.66 & $\mathrm{~A}$ \\
Bond & Lazer & 6.50 & 1.80 & $\mathrm{~A}$ \\
\hline
\end{tabular}

Not: Aynı harf gruplar arasında istatistiksel bir farkın olmadığını göstermektedir.

Tüm gruplara ait kırıma skorları Tablo 2' de verilmiştir. Mikroskop altında yapılan incelemelere göre örneklerdeki kırımaların daha çok amalgam ve adeziv arasında gerçekleştiği görülmüştür.

Tablo 2.

Gruplara ait kırılma başarısızlıklarının ARI skorlamalarına göre sonuçları

\begin{tabular}{cccccc|} 
Adezivler & Pürüzlendirme & $\mathbf{0}$ & $\mathbf{1}$ & $\mathbf{2}$ & $\mathbf{3}$ \\
\hline \multirow{2}{*}{$\begin{array}{c}\text { Transbond } \\
\text { XT }\end{array}$} & Frez & 10 & 0 & 0 & 0 \\
& Kumlama & 10 & 0 & 0 & 0 \\
\multirow{3}{*}{ Clearfil } & Lazer & 9 & 1 & 0 & 0 \\
& Kumlama & 10 & 0 & 0 & 0 \\
& Lazer & 10 & 0 & 0 & 0 \\
\hline
\end{tabular}




\section{TARTIŞMA}

Bu çalışmanın amacı, klinikte zaman zaman karşılaşılan molar dişlerdeki bukkal yüzeyleri kaplayan büyük amalgam dolgulu yüzeylere ortodontik amaçlı aparatların en iyi şekilde bağlantısını sağlayacak yüzey hazırlama metodunu ve uygun adeziv materyalini belirlemektir. $\mathrm{Bu}$ amaçla yapılmış olan araştırmamızda test edilen tüm yüzey hazırlama yöntemlerinin, iki farklı tip adeziv sistemi ile yapılan bağlanma dayanımı değerlerinin karşılaşıırmasında istatiksel olarak anlamlı bir farklılık olmadığı gözlenmiştir. Lazer ile yapılan yüzey pürüzlendirme prosedürü diğer iki gruptan daha yüksek değerler göstermiş olmasına rağmen bu değerler istatistiksel olarak anlamlı bulunmamıştır. Bu çalışmanın sonuçlarına göre amalgam yüzeylerinde frez ile oluşturulan pürüzlendirme metodunun klinikte hem uygulanışı kolay ve ucuz bir yöntem olması, hem de ortodontik molar tüplerin bu yüzeylere klinik olarak yeterli bağlanma dayanımına sahip olması açısından uygun bir yöntem olduğu görülmektedir.

Günümüzde braketlerin mine yüzeylerine bağlanma gücünü artırmak için farklı yüzey hazırlama teknikleri (mekanik, kimyasal) ya da farklı adeziv sistemlerle yapılan çeşitli çalışmalarla olumlu sonuçlar elde edildiği görülmüştür . ${ }^{8}$

Ortodontik klinik uygulamalarda posterior bölgede ulaşılması zor olan aynı zamanda restorasyonlu (kompozit, cam iyonomer veya amalgam) bukkal yüzeyleri bulunan molar dişlere tüplerin bağlanmasıyla ilgili ciddi sorunlar yaşanmaktadır. Bu sorunları çözmek amacıyla literatürde, kompozit ya da amalgam yüzeylere ortodontik braketlerinin bağlanması üzerine farklı yüzey uygulamalarının, farklı yapıştııcı simanların ve adeziv sistemlerin etkinliği ile ilgili bazı çalışma sonuçları bulunmaktadır. ${ }^{10,6,8,4}$

Erdemir ve ark. (2005) amalgamın tamirinde farklı adezivlerin başarısını araştırmışlar ve iki basamaklı selfetch adezivlerin daha başarılı bağlanma dayanımı sergilediğini rapor etmişlerdir. ${ }^{5}$ Bizim çalışmamızda biri geleneksel ortodontik adezivsistem olan Transbond XT ve diğeri ise dual-cure amalgabond adeziv sistem olan Clearfil Universal bond olmak üzere iki farklı adeziv sistem kullanıldı. Çalışmanın sonucunda adeziv sistemlerin farklı yüzey uygulamaları arasında bağlanma dayanımları açısından benzer sonuçlar elde edildi. Bayram ve ark. (2011) bir çalışmalarında yaşlandırılmış kompozit yüzeylere uyguladıkları farkı yüzey düzenleyici uygulamaların, ortodontik braketlere bağlanma dayanımları üzerine etkilerini araştırmışlar ve bu çalışmanın sonucunda tüm yüzey düzenleyici uygulamaların klinik için yeterli ortodontik bağlanma dayanımı oluşturabileceğini bildirmişlerdir. ${ }^{1}$ Yapılan bir çalışmada gümüşlü amalgam yüzeyine deney grubu olarak kumlama ve Er,Cr:YSGG lazer uygulanmış, amalgam yüzeylerine hiç bir şey uygulanmayan bir grupta kontrol grubu olarak değerlendirilmiştir. Çalışmanın sonuçlarına göre Er,Cr:YSGG lazerin braketlerin amalgam yüzeylere bağlantısını artırdığı rapor edilmiştir. ${ }^{8}$ Çalışmamızda amalgam yüzeylere uygulanan frez ile düzenleme, kumlama ve Er:YAG lazer uygulamaları arasında benzer bağlanma dayanım sonuçları elde edilmiştir. Diğer çalışmadan farklı olarak yaptığımız çalışmada Er:YAG lazer kullanılmıştır. Bu nedenle sonuçlardaki farklıı̆ı̆ da kullandığımız lazerden ve uygulama modlarındaki farklılıktan kaynaklandığı düşünülmektedir.

Germeç ve ark. (2009) amalgam yüzeylere ortodontik braketlerin bağlantısını, geleneksel ortodontik bonding sistem, bir amalgabond adeziv sistem ve ara bağlayıcı rezin olan üç farklı sistem kombinasyonlarının bağlanma dayanım değerlerini, kendi aralarında ve asitlenmiş mine yüzeyine uygulanan 2 farklı adeziv sistemin bağlanma dayanım değerleri ile karşılaştırmışlar, sonucunda asitlenmiş mine yüzeylerine uygulanan adeziv sistemlerin en yüksek bağlantı değerleri sağladığını gözlemlemişlerdir. ${ }^{6}$ Ayrıca yaptıkları çalışmada grupların kırıma başarısızıklarını değerlendirmişler ve kırılma başarısızlıklarının adeziv ara yüzeylerden olduğunu saptamışlardır. Bizim çalışmamızda da benzer olarak bir geleneksel ortodontik adeziv sistem bir de amalgabond adeziv sistem kullanılımış ancak aralarında bütün yüzey uygulamaları için bağlanma dayanımı açısından herhangi bir farklılık saptanmamıştır. Ayrıca bizim çalışmamızda da grupların kırılma başarısızlıklarının adeziv ara yüzeylerden yüzeylerde bozulmaya neden olmayacak şekilde olduğu gözlenmiştir.

\section{SONUÇ}

$\mathrm{Bu}$ çalışmanın limitasyonları dahilinde amalgam yüzeyine yapılan tüm mekanik düzenlemelerin ortodontik metal braket veya molar tüplerin bağlantısında klinik olarak benzer bağlanma dayanımı oluşturdukları ve kullanılan hem geleneksel ortodontik adeziv sistemin hem de amalgabond adeziv sistemin amalgam yüzeylere uygulanan tüm yüzey uygulamalarında bağlanma dayanımı açısından benzer sonuçlar oluşturduğu gözlenmiştir. Bu çalışmanın sınırlamaları yapılan amalgam yüzeylerin klinikteki şartları tam sağlayamayan yaşlandırıımış amalgam yüzeyler olmamasıdır. Bu nedenle ileriki çalışmalarda farkı adeziv sistemlerin yaşlandırılmış amalgam yüzeylere yapıştıılan ortodontik braketler ve tüplerin bağlanma dayanımlarının da araştırılmasının gerektiğini düşünmekteyiz. 


\section{KAYNAKLAR}

1. Bayram M, Yesilyurt C, Kusgöz A, Ulker M, Nur $M$. Shear bond strength of orthodontic brackets to aged resin composite surfaces: effect of surface conditioning. Eur J Orthod 2011; 3(2): 174-9.

2. Buyukyilmaz $\mathrm{T}$, Zachrisson BU. Improved orthodontic bonding tosilver amalgam. part 2 . Lathe-cut, admixed, and spherical amalgams with different intermediate resins. Angle Orthod 1998; 68(4): 337-44.

3. Cernavin I, Hogan SP. The effects of theNd:YAG laser on amalgam dental restorative material. Aust Dent J 1999; 44(2): 98-102.

4. Demirtas HK, Akin M, Ileri Z, Basciftci FA. Shearbond-strength of orthodontic brackets to aged nano-hybrid composite-resin surfaces using different surface preparation. Dent Mater J 2015; 34(1): 86-90.

5. Erdemir A, Ünverdi A, Arı H, Belli S. Farklı Bonding Sistemlerin Kompozit Bezinin Amalgama Bağlanma Dayanımı Üzerine Etkisi Ondokuz Mayıs Univ. Diş Hekim Fak Derg 2005; 6(1): 9-13.

6. Germec D, Cakan U, Ozdemir FI, Arun T, Cakan $M$. Shear bond strength of brackets bonded to amalgam with different intermediate resins and adhesives. Eur J Orthod 2009; 31(2): 207-12.

7. Hossain M, Nakamura $Y$, Yamada $Y$, Suzuki N, Murakami Y, Matsumoto K. Analysis of surface roughness of enamel and dentin after Er,Cr:YSGG laser irradiation. J Clin Laser Med Surg 2001; 19(6): 297-303.

8. Oskoee PA, Kachoei M, Rikhtegaran S, Fathalizadeh F, Navimipour EJ. Effect of surface treatment with sandblasting and Er,Cr:YSGG laser on bonding of stainless steel orthodontic brackets to silver amalgam. Med Oral Patol Oral Cir Bucal 2012; 17(2): 292-6.

9. Pioch T, Matthias J. Mercury vapor release from dental amalgam after laser treatment. Eur $\mathrm{J}$ Oral Sci 1998; 106(1): 600-02.

10. Sperber RL, Watson PA, Rossouw PE, Sectakof PA. Adhesion of bonded orthodontic attachments to dental amalgam: In vitro study. Am J Orthod Dentofacial Orthop 1999;116(5): 506-13.

11. Usumez A, Aykent $F$. Bond strengths of porcelain laminate veneers to tooth surfaces prepared with acid and Er,Cr:YSGG Laser etching. J ProsthetDent 2003; 90(1): 24-30.

12. Yetkiner E, Özcan M. Adhesive strength of metal brackets on existing composite, amalgam and restoration-enamel complex following airabrasion protocols. Int J Adhes Adhes 2014; 54: 200-05.
13.Zachrisson BU, Buyukyilmaz T, Zachrisson YO. Improving orthodontic bonding to silver amalgam. Angle Orthod 1995; 65(1): 35-42.

Yazışma Adresi:

Yrd.Doç.Dr. Ahmet Ertan SOĞANCI

Necmettin Erbakan Üniversitesi

Diş Hekimliği Fakültesi Ortodonti AD

Karaciğan Mah. Ankara Cad. No: 74/A

PK 42050 Karatay, Konya

Tel : +90 3322200026

E-mail: esoganci@konya.edu.tr 